\title{
Evaluation of nilotinib in advanced GIST previously treated with imatinib and sunitinib
}

\author{
C. Cauchi $\cdot$ N. Somaiah $\cdot$ P. F. Engstrom $\cdot$ S. Litwin $\cdot$ \\ M. Lopez $\cdot$ J. Lee $\cdot$ M. Davey $\cdot$ B. Bove $\cdot$ M. von Mehren
}

Received: 10 June 2011 / Accepted: 8 November 2011 / Published online: 27 November 2011

(C) The Author(s) 2011. This article is published with open access at Springerlink.com

\begin{abstract}
Purpose Patients with advanced GIST following standard imatinib and sunitinib often have good performance status and need additional therapy. This study tested nilotinib, a second-generation tyrosine kinase inhibitor, in patients with advanced GIST refractory to standard therapies.

Methods This single-center open-label phase II study has a primary objective to determine progression-free survival at 6 months. Using a novel statistical design, 17 patients were to be enrolled; if $\geq 10$ were progression free (PF) at 2 months, 19 additional patients would be enrolled. The therapy was considered of benefit if $\geq 13$ of 36 patients were PF at 6 months. All patients signed informed consent and entry criteria included normal cardiac function. Exploratory analyses correlating genotype with response were also performed.

Results Thirteen patients were treated; 2 had received agents after imatinib and sunitinib. Treatment was well
\end{abstract}

C. Cauchi · M. Lopez

Regina Elena Institute for Cancer Research, Rome, Italy

N. Somaiah · P. F. Engstrom · M. von Mehren $(\varangle)$

Department of Medical Oncology, Fox Chase Cancer Center, Philadelphia, PA, USA

e-mail: Margaret.vonMehren@fccc.edu

S. Litwin

Department of Biostatistics, Fox Chase Cancer Center, Philadelphia, PA, USA

J. Lee $\cdot$ M. Davey

Protocol Management Office, Fox Chase Cancer Center,

Philadelphia, PA, USA

B. Bove

Department of Molecular Pathology, Fox Chase Cancer Center, Philadelphia, PA, USA tolerated with one grade 4 anemia attributed to nilotinib. No measurable responses were observed; median time to progression was 2 months. One patient remained on study with stable disease for 12 months. Mutation testing is available from 10 primary tumors with 7 exon 11 mutations, 1 exon 9 mutation, and 2 without KIT/PDGFR mutations. Two samples from recurrent disease had 2 mutations, both primary exon 11 mutations with an additional exon 17 mutation, including the patient with prolonged stable disease.

Conclusions Nilotinib was well tolerated in these patients with advanced GIST. Accrual was halted due to insufficient clinical benefit. However, nilotinib may provide benefit to specific subsets of advanced GIST with exon 17 mutations.

Keywords GIST - Tyrosine kinase inhibitor · Nilotinib · Kit mutations $\cdot$ Secondary mutations

\section{Introduction}

Imatinib, a selective inhibitor of several tyrosine kinases, including ABL, ARG, KIT, and PDGFRA/B, has significantly improved the clinical outcome of patients with advanced GIST. Treatment with imatinib may be of clinical benefit of $80-90 \%$ of patients with advanced or metastatic GIST, with a median time to progression of 2 years $[1,2]$. Sunitinib, an oral multitargeted tyrosine kinase inhibitor with activity against KIT, PDGFR, VEGFR, and FLT$1 / \mathrm{KDR}$, is utilized in the second-line setting. A phase III [3] double-blind placebo-controlled trial testing sunitinib showed that the time to progression was significantly longer in the sunitinib group than in the placebo group (6.3 vs. 1.5 months). However, despite initial response or stabilization of the disease, secondary resistance develops in 
most patients after approximately 7 months. Patients progressing following standard imatinib and sunitinib often still have good performance status and need additional therapy.

This study was undertaken to evaluate the benefit of nilotinib, a second-generation tyrosine kinase inhibitor that functions via ATP-competitive inhibition. Nilotinib was developed to optimize binding to bcr-abl for the therapy of chronic myelogenous leukemia. In vitro, it can inhibit KIT and PDGFR phosphorylation, has clinical activity against imatinib-resistant cell lines, and achieves higher intracellular concentrations compared to imatinib. Data from a phase I study [4] suggest that nilotinib is well tolerated and has clinical activity in patients with imatinib-resistant GIST who have failed second-line targeted therapies.

\section{Patients and methods}

\section{Study objectives and design}

This was a single-center open-label phase II study. The primary endpoint was to establish the progression-free survival (PFS) rate at 6 months in patients receiving nilotinib, who had been previously treated with at least imatinib and sunitinib. The secondary endpoint was to determine the response rate to nilotinib. An exploratory endpoint correlating genotype with response was also performed.

Patients with pathologically confirmed advanced GIST were eligible for this study if they met the following key criteria: age $\geq 18$ years, failure of treatment with imatinib and sunitinib, measurable disease to RECIST criteria [12], an ECOG performance status (PS) of 0-2, a life expectancy of greater than 12 weeks, and adequate major organ function. Key exclusion criteria included abnormal cardiac function, chronic liver disease, and other severe and/or uncontrolled concurrent medical diseases. The study was approved by the Institutional Review Board of Fox Chase Cancer Center, and written informed consent was obtained from all patients.

Nilotinib was administered orally (fasting) at a dose of $400 \mathrm{mg}$ BID, every $12 \mathrm{~h}$ continuously. All patients were scheduled to continue treatment until disease progression or unacceptable toxicity. Patients had regular physical examination, evaluation of performance status, complete blood count, serum chemistry, and assessment of toxic effects and tumor response. Tumor evaluation was performed at baseline and every 8 weeks thereafter by CT scan of chest, abdomen, and pelvis or MRI of abdomen and pelvis, and CT scan of chest. Objective tumor response was defined according to RECIST [5]. In this study, the objective tumor response was also evaluated utilizing $\mathrm{CHOI}$ criteria [6] as well, to obtain data on the role of CHOI criteria to assess disease response in patient with advanced GIST refractory to imatinib and sunitinib.

Progression-free survival, the primary endpoint, was defined as the duration of time from start of the treatment to time of progression or death, according to RECIST criteria. Toxicity was graded according to the National Cancer Institute Common Toxicity Criteria (CTCAE Version 3.0).

Molecular testing at Fox Chase Cancer Center for the determination of KIT and PDGFRA mutations was performed if tumor samples from the primary tumor resection were available. Blood samples were collected from all patients at baseline and at the time of the first tumor assessment for potential further study.

\section{Statistical design}

The study tested the composite null hypothesis that the chance of PFS to 2 months is at most $60 \%$ and the chance of PFS to 6 months is at most $25 \%$ utilizing a new phase II design that allows evaluation for futility both early in patient numbers and early in the course of treatment [7]. An initial 17 patients were to be entered; if at least $11 / 17$ were $\mathrm{PF}$ at 2 months, an additional 19 patients were to be added. Patients from the initial cohort who are PFS at 2 months continue on study while PF and are followed for 6 months as are the 19 new patients. If the number of PF patients from both initial and second cohorts at 6 months is at least 13/36, we will reject the null hypothesis. The study has $90.4 \%$ overall power and $3.4 \%$ overall type I error. The chance of stopping the study early in a treatment arm is 55\% under the null hypothesis. The chance of stopping early in error in any treatment arm (when the chances are $80 \%$ and $50 \%$ for 2 month and 6 month PFS, respectively) is $4 \%$. Therefore, for the standard phase II design, up to 36 patients will be accrued.

\section{Results}

Patients

Between July 2008 and July 2009, 15 patients were signed consent for this study. Two patients were judged to be ineligible: One patient due to a cardiac arrhythmia identified during screening. A second patient with a history of GIST diagnosed at another institution began therapy having previously received imatinib and sunitinib with evidence of response; pathology review diagnosed a malignant fibrous tumor, and mutational testing identified no KIT or PDGFRA mutations. Thirteen patients were treated as per protocol and are evaluable for response and toxicity evaluation. Characteristics of the patients are summarized in Table 1. The median age was 63 years (range 54-78), and the median ECOG performance status was 1 (range $0-1$ ). Primary 
Table 1 Patient demographics $(n=13)$

\begin{tabular}{|c|c|}
\hline \multicolumn{2}{|l|}{ Age in years } \\
\hline Median & 63 \\
\hline \multirow[t]{2}{*}{ Range } & $54-78$ \\
\hline & $n(\%)$ \\
\hline \multicolumn{2}{|l|}{ Sex } \\
\hline Male & $9(69.2)$ \\
\hline Female & $4(30.8)$ \\
\hline \multicolumn{2}{|l|}{ Race } \\
\hline White & $10(77)$ \\
\hline Black & $3(23)$ \\
\hline \multicolumn{2}{|l|}{ ECOG performance status } \\
\hline 0 & $4(30.8)$ \\
\hline 1 & $9(69.2)$ \\
\hline 2 & 0 \\
\hline \multicolumn{2}{|l|}{ Primary site of tumor } \\
\hline Stomach & $6(46.1)$ \\
\hline Small bowel & $5(38.5)$ \\
\hline Colon & $1(7.7)$ \\
\hline Rectum & $1(7.7)$ \\
\hline \multicolumn{2}{|l|}{ Metastatic sites } \\
\hline Liver & $11(84.6)$ \\
\hline Peritoneum/abdomen & $7(53.8)$ \\
\hline Pelvis & $4(30.8)$ \\
\hline Lung & $2(15.4)$ \\
\hline Spleen & $1(7.7)$ \\
\hline Bone & $1(7.7)$ \\
\hline Lymph node & $1(7.7)$ \\
\hline
\end{tabular}

tumor site was stomach $(n=6)$, small bowel $(n=5)$, and colon/rectum $(n=2)$, while the most common metastatic sites were liver and peritoneum. All patients had received prior imatinib and sunitinib; two patients had received prior medical therapy beyond imatinib and sunitinib.

Tolerability

Treatment was generally well tolerated. Side effects attributed to nilotinib are listed in Table 2. The most common adverse events included fatigue (100\%), anorexia $(61 \%)$, constipation (54\%), anemia (92\%), and leucopenia (54\%). Additional side effects included were GI related: diarrhea $(38 \%)$, nausea $(38 \%)$, abdominal pain (38\%); dyspnea $(38 \%)$, elevated alkaline phosphatase $(46 \%)$, hypoalbuminemia (46\%), and electrolyte abnormalities: hyperkalemia $(38 \%)$, hyponatremia (30\%), and hypomagnesemia (30\%). Most of these adverse events were mild or moderate (grade 1 or 2). Side effects of grade 3 occurred in $23 \%$ of patients. One patient had a grade 4 anemia. The median exposure to nilotinib was 42.5 days (range $21.5-372$ ).
Dose modifications

Dose modifications were utilized for management of toxicities as specified in the protocol. Five patients $(42 \%)$ required drug to be held. One patient $(8 \%)$ required a dose reduction for a rise in creatinine that occurred in the setting of hospitalization for pneumonia. After 8 weeks at $400 \mathrm{mg}$ daily, the dose was escalated back to $400 \mathrm{mg}$ BID as the creatinine returned to baseline where it remained. The overall dose density was $93.4 \%$, with seven patients (58\%) taking all prescribed doses.

\section{Efficacy}

Accrual was halted after 13 eligible patients enrolled due to insufficient clinical benefit, since 8 of 13 patients progressed by 2 months. Assessment of response was made utilizing both RECIST and CHOI criteria. Data on antitumor response are shown in Table 3. Of the thirteen patients entered on study, 12 had follow-up imaging to assess response. There were no complete or partial responses by RECIST, with 4 patients achieving stable disease, one of which lasted 12 months.

\section{Disease assessment by CHOI}

Eight of the 12 patients were able to have response determined by CHOI criteria. One patient had stable disease by both criteria, one patient progressive disease by RECIST and stable disease by CHOI, a third patient had stable disease by RECIST and response by $\mathrm{CHOI}$, and the remaining 5 patients all had progressive disease by both criteria (see Table 3). Of note, the patient with the response by CHOI was taken off study for clinical progression.

Molecular testing results

Of 10 patients who underwent molecular testing of primary tumor samples, KIT exon 11 and 9 mutations were observed in five and one patients, respectively. The results are summarized in Table 4. Of interest, two samples demonstrated two mutations: a primary KIT exon 11 mutation with KIT secondary exon 17 mutation, which included the patient with prolonged stable disease. A correlation between type of mutation and clinical outcome is shown in Table 4.

\section{Discussion}

The use of tyrosine kinase inhibitors has revolutionized the management of GIST, significantly improving patient outcomes. At present, patients progressing on first-line imatinib treatment have the possibility of imatinib dose 
Table 2 Toxicities attributable to nilotinib

\begin{tabular}{|c|c|c|c|c|c|}
\hline \multirow[t]{2}{*}{ Adverse event $(n=13)$} & \multicolumn{5}{|c|}{ Grade (NCI-CTCAE version 3.0), $n(\%)$} \\
\hline & 1 & 2 & 3 & 4 & All grades \\
\hline Anemia & $6(46)$ & $5(38.5)$ & 0 & $1(7.7)$ & $12(92)$ \\
\hline Leukopenia & $6(46)$ & $1(7.7)$ & 0 & 0 & $7(54)$ \\
\hline Neutropenia & $3(23)$ & 0 & 0 & 0 & $3(23)$ \\
\hline Thrombocytopenia & 0 & 0 & 0 & 0 & 0 \\
\hline Fatigue & $12(92)$ & $1(7.7)$ & 0 & 0 & $13(100)$ \\
\hline Diarrhea & $5(38.5)$ & 0 & 0 & 0 & $5(38.5 \%)$ \\
\hline Constipation & $4(30.8)$ & $2(15)$ & $1(7.7)$ & 0 & $7(54)$ \\
\hline Rash & $5(38.5)$ & 0 & 0 & 0 & $5(38.5)$ \\
\hline Pruritus & $1(7.7)$ & 0 & 0 & 0 & $1(7.7)$ \\
\hline Edema & $1(7.7)$ & $2(15)$ & 0 & 0 & $3(23)$ \\
\hline Nausea & $5(38.5)$ & 0 & 0 & 0 & $5(38.5)$ \\
\hline Vomiting & $2(15)$ & 0 & 0 & 0 & $2(15)$ \\
\hline Anorexia & $8(61)$ & 0 & 0 & 0 & $8(61)$ \\
\hline Abdominal pain & $4(30.8)$ & 0 & $1(7.7)$ & 0 & $5(38.5)$ \\
\hline Headache & 0 & 0 & 0 & 0 & 0 \\
\hline Bone pain & 0 & $1(7.7)$ & 0 & 0 & $1(7.7)$ \\
\hline Voice change & $4(30.8)$ & 0 & 0 & 0 & $4(30.8)$ \\
\hline Dyspnea & $4(30.8)$ & $1(7.7)$ & 0 & 0 & $5(38.5)$ \\
\hline Cough & $2(15)$ & 0 & 0 & 0 & $2(15)$ \\
\hline Arthralgia/myalgia & $4(30.8)$ & 0 & 0 & 0 & $4(30.8 \%)$ \\
\hline Infection & 0 & $1(7.7)$ & 0 & 0 & $1(7.7)$ \\
\hline Elevated creatinine & $3(23)$ & $1(7.7)$ & 0 & 0 & $4(30.8)$ \\
\hline Transaminitis & $3(23)$ & $1(7.7)$ & 0 & 0 & $4(30.8)$ \\
\hline Elevated bilirubin & $2(15)$ & 0 & 0 & 0 & $2(15)$ \\
\hline Elevated alkaline phosphatase & $4(30.8)$ & $1(7.7)$ & $1(7.7)$ & 0 & $6(46)$ \\
\hline Elevated amylase/lipase & $2(15)$ & 0 & 0 & 0 & $2(15)$ \\
\hline Hypoalbuminemia & $4(30.8)$ & $2(15)$ & 0 & 0 & $6(46)$ \\
\hline Hyponatremia & $4(30.8)$ & 0 & 0 & 0 & $4(30.8)$ \\
\hline Hyperkalemia & $5(38.5)$ & 0 & 0 & 0 & $5(38.5)$ \\
\hline Hypocalcemia & $1(7.7)$ & 0 & 0 & 0 & $1(7.7)$ \\
\hline Hypomagnesemia & $4(30.8)$ & 0 & 0 & 0 & $4(30.8)$ \\
\hline
\end{tabular}

Table 3 Best tumor response as determined by RECIST and by CHOI criteria, $n=13$

\begin{tabular}{lll}
\hline Response & RECIST/n(\%) & $\mathrm{CHOI}^{\mathrm{a}} / n(\%)$ \\
\hline $\mathrm{CR}$ & 0 & 0 \\
$\mathrm{PR}$ & 0 & $1^{\mathrm{b}}(8)$ \\
$\mathrm{SD}$ & $4(31)$ & $2^{\mathrm{b}}(15)$ \\
$\mathrm{PD}$ & $8(62)$ & $5(38)$ \\
$\mathrm{NE}$ & $1(8)$ & $5(38)$ \\
\hline
\end{tabular}

${ }^{\text {a }}$ CHOI responses were assessable in 8 patients only, due to the use of MRI or CT without IV contrast imaging in 4 patients, and one patient was inevaluable due to lack of follow-up imaging

${ }^{b}$ CHOI PR was SD by RECIST. The CHOI SD were evaluated as SD and PD by RECIST escalation or treatment with sunitinib. However, despite initial response or stabilization, secondary resistance develops in most of the patients. The acquired resistance to imatinib and sunitinib represents a major clinical challenge, and this phase II study tested the benefit of nilotinib in patients, that had failed treatment with at least imatinib and sunitinib, and the correlation between clinical outcome and mutational status of the tumor.

Nilotinib administered as a single agent was well tolerated, confirming other reports in GIST patients. Accrual of patients was halted due to insufficient clinical benefit. No patient had a complete or a partial response, and a stable disease was observed in $30 \%$ of patients. It should be noted that objective responses in GIST patients that have 
Table 4 Correlation of mutation with clinical outcome on nilotinib

$P D$ progressive disease; $S D$ stable disease

\begin{tabular}{ll}
\hline Type of mutation & Clinical outcome \\
\hline KIT exon 11-1691del6 at amino acid 556 & PD after 3 cycles \\
KIT exon 11-alteration begins at K550 & SD after 2 cycles \\
KIT exon 11-alteration begins at Q556 & PD post 2 cycles \\
KIT exon 11-V599D & PD after 3 cycles \\
KIT exon 11-L576P & SD post 2 cycles \\
KIT exon 11-alteration begins at K558 and KIT exon 17-N822 K & PD post 2 cycles \\
KIT exon 11-alteration begins at V555 and KIT exon 17-Y823D & PD after 12 cycles \\
KIT exon 9 mutation-A502_Y503del & PD post 2 cycles \\
No identifiable mutation in KIT or PDGFRA & PD post 2 cycles \\
No identifiable mutation in KIT or PDGFRA & PD post 2 cycles \\
\hline
\end{tabular}

progressed on standard therapies are uncommon, and thus the lack of CR or PR is not surprising. Median progressionfree survival was 2 months.

In contrast to other reports of the benefit of nilotinib in patients with advanced GIST, the progression-free survival in our study was shorter than that reported elsewhere. A retrospective European analysis of 52 patients treated with nilotinib showed objective response in $10 \%$ of patients $(95 \%$ CI 2-18); 37\% (95\% CI 24-50) had disease stabilization. Median PFS and OS were 12 weeks (95\% CI 9-15) and 34 weeks (95\% CI 3-65), respectively [8]. In a phase II Japanese study, 35 patients were treated with nilotinib. Median PFS was 113 days and median OS was 310 days; disease control rate at 24 weeks was $28.6 \%$ (90\% CI 16.4$43.6 \%$ ); $65.7 \%$ of patients had a stable disease, and $2.9 \%$ had a partial response [9]. In a Korean study, 2 of 17 patients, treated with nilotinib, had a partial response and 10 of 17 had a stable disease with a clinical benefit rate at 24 weeks of $47 \%$. Median PFS and OS were 23.6 weeks (95\% CI 0-50.6 weeks) and 74 weeks (95\% CI 27.4120.6 weeks), respectively [10]; intriguingly this study evaluated drug levels and noted lower serum levels of nilotinib in patients with gastric resections, particularly those with complete resections. In our study, one patient had had a prior total gastrectomy (PD after 1 cycle) and an additional 3 patients had partial or hemigastrectomies (PD after 2-3 cycles). Lastly, the phase III trial of Nilotinib compared with supportive care, including the use of imatinib or sunitinib, did not demonstrate a significant progression-free or overall survival benefit in a similar study population; there did appear, however, to be a progression benefit in those patients who entered on the study after therapy only with imatinib and sunitinib [11]. Our statistical assumptions lead to early termination of our accrual and thus may have limited our power to detect a benefit of nilotinib.

The correlation between clinical outcome and tumor genotype is of interest. Secondary mutations in exons 13 and 14 are known to be well controlled by sunitinib; however, those in exon 17 are not [12]. The patient that remained on study for 12 cycles had a biopsy prior to study entry that demonstrated a primary KIT exon 11 (alteration beginning at K558) with a secondary exon 17 mutation (Y823D). In vitro studies by Guo and colleagues suggested that Nilotinib in cell lines transfected with a KIT gene containing an exon 9 or 11 mutation with an exon 17 mutation (D820Y and $\mathrm{N} 822 \mathrm{~K}$ ) demonstrated significant kinase inhibition as well as decreased cell proliferation The Japanese study also documented a partial response in patients whose GIST contained a KIT exon 11 (dup 567-576) and exon 17 (D820G) mutation [9]. The benefit of nilotinib for patients with exon 11 and 17 mutations is intriguing but appears to be dependent on the specific secondary mutation in exon 17 . In addition, other cellular factors beyond the mutations may play a role in whether a response is noted, as is exemplified by our second patient with the exon 11 (alteration beginning at 558) and exon 17 (N822 K), who would have been predicted to have benefit from nilotinib based on in vitro data, but whose tumor progressed after 2 months of therapy.

We concluded that nilotinib was generally well tolerated and may provide benefit to specific subset of advanced GIST with secondary KIT exon 17 mutations, a group of patients that have no standard therapeutic options. Further studies are needed to confirm these data and to define the role of nilotinib for treatment of GIST, alone or in combination with other drugs.

Acknowledgments The investigators would like to thank the patients who participated in this study and their families. This study was an investigator-initiated trial supported in part by Novartis Pharmaceuticals. It was also supported by our institutional core grant, $\mathrm{NCI}$ CA06927, as well as NCI R01 CA106588 (MvM).

Conflict of interest MvM serves as a scientific advisor to Novartis.

Open Access This article is distributed under the terms of the Creative Commons Attribution Noncommercial License which permits any noncommercial use, distribution, and reproduction in any medium, provided the original author(s) and source are credited. 


\section{References}

1. Demetri GD, von Mehren M, Blanke CD, Van den Abbeele AD, Eisenberg B, Roberts PJ, Heinrich MC, Tuveson DA, Singer S, Janicek M, Fletcher JA, Silverman SG, Silberman SL, Capdeville R, Kiese B, Peng B, Dimitrijevic S, Druker BJ, Corless C, Fletcher CD, Joensuu H (2002) Efficacy and safety of Imatinib mesylate in advanced gastrointestinal stromal tumors. N Engl J Med 347:472480

2. Blanke CD, Demetri GD, von Mehren M, Heinrich MC, Eisenberg B, Fletcher JA, Corless CL, Fletcher CD, Roberts PJ, Heinz D, Wehre E, Nikolova Z, Joensuu H (2008) Long-term results from a randomized phase II trial of standard- versus higher-dose imatinib mesylate for patients with unresectable or metastatic gastrointestinal stromal tumors expressing KIT. J Clin Oncol 26:620-625

3. Demetri GD, van Oosterom AT, Garrett CR, Blackstein ME, Shah MH, Verweij J, McArthur G, Judson IR, Heinrich MC, Morgan JA, Desai J, Fletcher CD, George S, Bello CL, Huang X, Baum CM, Casali PG (2006) Efficacy and safety of sunitinib in patients with advanced gastrointestinal stromal tumor after failure of imatinib: a randomized controlled trial. Lancet 368:1329-1338

4. Demetri GD, Casali PG, Blay JY, von Mehren M, Morgan JA, Bertulli R, Ray-Coquard I, Cassier P, Davey M, Borghaei H, Pink D, Debiec-Rychter M, Cheung W, Bailey SM, Veronese ML, Reichardt A, Fumagalli E, Reichardt P (2009) A phase I study of single-agent nilotinib or in combination with imatinib in patients with imatinib-resistant gastrointestinal stromal tumors. Clin Cancer Res 15:5910-5916

5. Therasse P, Arbuck SG, Eisenhauer EA, Wanders J, Kaplan RS, Rubinstein L, Verweij J, Van Glabbeke M, van Oosterom AT, Christian MC, Gwyther SG (2000) New guidelines to evaluate the response to treatment in solid tumors. J Natl Cancer Inst 92:205216

6. Choi H, Charnsangavej C, Faria SC, Macapinlac HA, Burgess MA, Patel SR, Chen LL, Podoloff DA, Benjamin RS (2007)
Correlation of computed tomography and positron emission tomography in patients with metastatic gastrointestinal stromal tumor treated at a single institution with imatinib mesylate: proposal of new computed tomography response criteria. J Clin Oncol 25:1753-1759

7. Litwin S, Wong YN, Hudes G (2007) Early stopping design based on progression-free survival at an early time point in the initial cohort. Stat Med 26:4400-4415

8. Montemurro M, Schöffski P, Reichardt P, Gelderblom H, Schütte J, Hartmann JT, von Moos R, Seddon B, Joensuu H, Wendtner CM, Weber E, Grünwald V, Roth A, Leyvraz S (2009) Nilotinib in the treatment of advanced gastrointestinal stromal tumours resistant to both imatinib and sunitinib. Eur J Cancer 45:22932297

9. Nishida T, Sawaki A, Doi T, Yamada Y, Komatsu Y, Kanda T, Kakeji Y, Onozawa Y, Yamasaki M, Ohtsu A (2010) Phase II trial of nilotinib as third-line therapy for gastrointestinal stromal tumor (GIST) patients in Japan. J Clin Oncol 28:15s (suppl; abstr 10015)

10. Kim KP, Ryu MH, Yoo C, Ryoo BY, Choi DR, Chang HM, Lee JL, Beck MY, Kim TW, Kang YK (2010) Nilotinib in patients with GIST who failed imatinib and sunitinib: importance of prior surgery on drug bioavailability. Cancer Chemother Pharmacol 2010 Oct 19 [Epub ahead of print]

11. Reichardt P, Blay JY, Gelderblom H, Schlemmer M, Demetri GD, Bin Bui N, McArthur GA, Yazji S, Hsu Y, Rutkowski P (2010) Phase III trial of nilotinib in patients with advanced gastrointestinal stromal tumor (GIST): First results from ENEST g3. J Clin Oncol 28:15s (suppl; abstr 10017)

12. Heinrich MC, Maki RG, Coreless C, Antonescu CR, Harlow A, Griffith D, Town A, McKinley A, Ou WB, Fletcher JA, Fletcher CD, Huang X, Cohen DP, Baum CM, Demetri GD (2008) Primary and secondary kinase genotypes correlate with the biological and clinical activity of sunitinib in imatinib-resistant gastrointestinal stromal tumor. J Clin Oncol 26:5352-5359 